Apidologie, 1976, 7 (2), 113-127.

\title{
RELATIONS INTERSPÉCIFIQUES ET INTRASPÉCIFIQUES ENTRE BUTINEUSES DE BOMBUS SP. ET D'APIS MELLIFICA L.
}

\author{
Interspezifische und intraspezifische Beziehungen bei den \\ Sammlerinnen von Bombus sp. und Apis mellifica $L$. \\ G. BENEST \\ Station de Zoologie, \\ Centre national de Recherches agronomiques, I.N.R.A., \\ 78000 Versailles
}

\section{SUMMARY}

INTERSPECIFIC AND INTRASPECIFIC RELATIONS BETWEEN FORAGERS of Bombus Sp. AND Apis mellifica L.

Pollen-feeding and nectar-feeding insects are numerous meeting on flowers : observed contacts are both intraspecific and interspecific. Behaviours are studied by observing workers of Apis mellifica, Bombus lapidarius, B. terrestris and B. agrorum foraging dwarf dahlias.

Data show that meetings are not due to chance : joint foraging of one flower is accepted by insects of the same species and avoided by insects of different species. Moreover honey bees better tolerate such joint foraging than bumble bees do. It is proposed that interindividual recognition is achieved through visual information.

\section{RÉSUMÉ}

Nombreux sont les insectes pollinivores et nectarivores à se rencontrer sur des fleurs : les contacts observés sont interspécifiques et intraspécifiques. L'étude des comportements est faite par l'observation d'ouvrières d'Apis mellifica, Bombus lapidarius, B. terrestris et $B$. agrorum butinant des dahlias nains.

Les résultats montrent que les rencontres ne sont pas dues au hasard : le butinage en commun d'une fleur est accepté par des insectes de même espèce, mais évité par des insectes d'espèces différentes. De plus les abeilles supportent mieux ce butinage en commun que les bourdons. Une hypothèse est proposée selon laquelle la reconnaissance interindividuelle serait faite au moyen de la vue. 


\section{INTRODUCTION}

L'étude des relations qui s'établissent entre les insectes butinant les mêmes fleurs, aboutit la plupart du temps à la constatation d'une influence importante d'un congénère sur une butineuse. Ainsi on sait que l'abeille fréquente avec assiduité certaines aires dites de butinage. Lecomte (1961) observe même les colonies, dans certaines circonstances, se réserver l'accès de ces aires et des chemins de vol qui y vont.

De tels phénomènes sont difficiles à observer chez Bombus car les colonies sont trop petites. Néanmoins, il est certain qu'une ouvrière de bourdon butine souvent la même aire.

A cet endroit, les rencontres intraspécifiques et interspécifiques sont nombreuses. Leur observation permet d'étudier les relations qui s'établissent entre deux insectes butinant les mêmes fleurs et ainsi aider à comprendre la façon dont ces animaux résolvent les problèmes de compétition.

\section{MATÉRIEL ET MÉTHOdES}

Les animaux sont des insectes sauvages vivant dans les environs de la Station de Recherches sur l'Abeille et les In-ectes Sociaux de Bures-sur-Yvette et venant butiner les fleurs cultivées à leur intention. Ce sont presque exclusivement des Bombus et des Apis mellifica.

$$
\text { 1. - Les fleurs }
$$

Il s'agit d'un champ de $4.72 \mathrm{~m}^{2}$ de dahlias nains, simples, soit jaunes, soit rouges. Les pieds sont espacés de 80 centimètres et alignés en 26 rangées distantes de 80 can.

\section{2. - Observations}

Tous les jours pendant 2 mois en 1972 et en 1973, des comptages ont été faits sur 8 des 26 rangées du champ. Ils sont faits à des heures variables au rythme de un par jour et dans des conditions climatiques permettant le vol des insectes.

\section{3. - Mesures}

La quantification est hasée sur la présence d'un insecte actif sur les 8 rangées. Ne sont comptés que les bourdons et abeilles, soit posés sur une fleur dont ils prennent le pollen ou le nectar soit, s'approchant d'une fleur occupée (c'est-à-dire jusqu'à une distance inférieure ou égale à $3 \mathrm{~cm}$ ). Dans ce cas, les comportements de l'arrivant et de l'occupant sont notés.

A chaque fleur butinée, il est noté l'espèce et le nombre des individus concernés.

Après chaque observation, les fleurs épanouies sont comptées, les fleurs fanées enlevées sur les 8 rangées.

\section{RÉSULTATS}

Au cours de ce travail, 7576 Bombinae (dont 7493 Bombus terrestris, B. lapidarius, B. agrorum) et 9153 Apis mellifica ont été vus. 
La plupart du temps, une fleur n'est visitée que par un seul insecte à la fois. Quelquefois, on peut voir 2, 3 animaux butiner le même dahlia. Un nombre plus grand de butineuses sur la même fleur est exceptionnel.

Le nombre de couples (c'est-à-dire 2 butineuses, quelle que soit leur espèce, sur une même fleur) est proportionnel à la densité $\left(\frac{\text { nombre d'insectes }}{\text { nombre de fleurs }}\right)$ d'individus sur le champ. Dans la figure 1 , tous les Bombus sont groupés et opposés à Apis mellifica. Les deux genres montrent une augmentation régulière du nombre de couples, parallèle à une augmentation de la densité du genre correspondant.

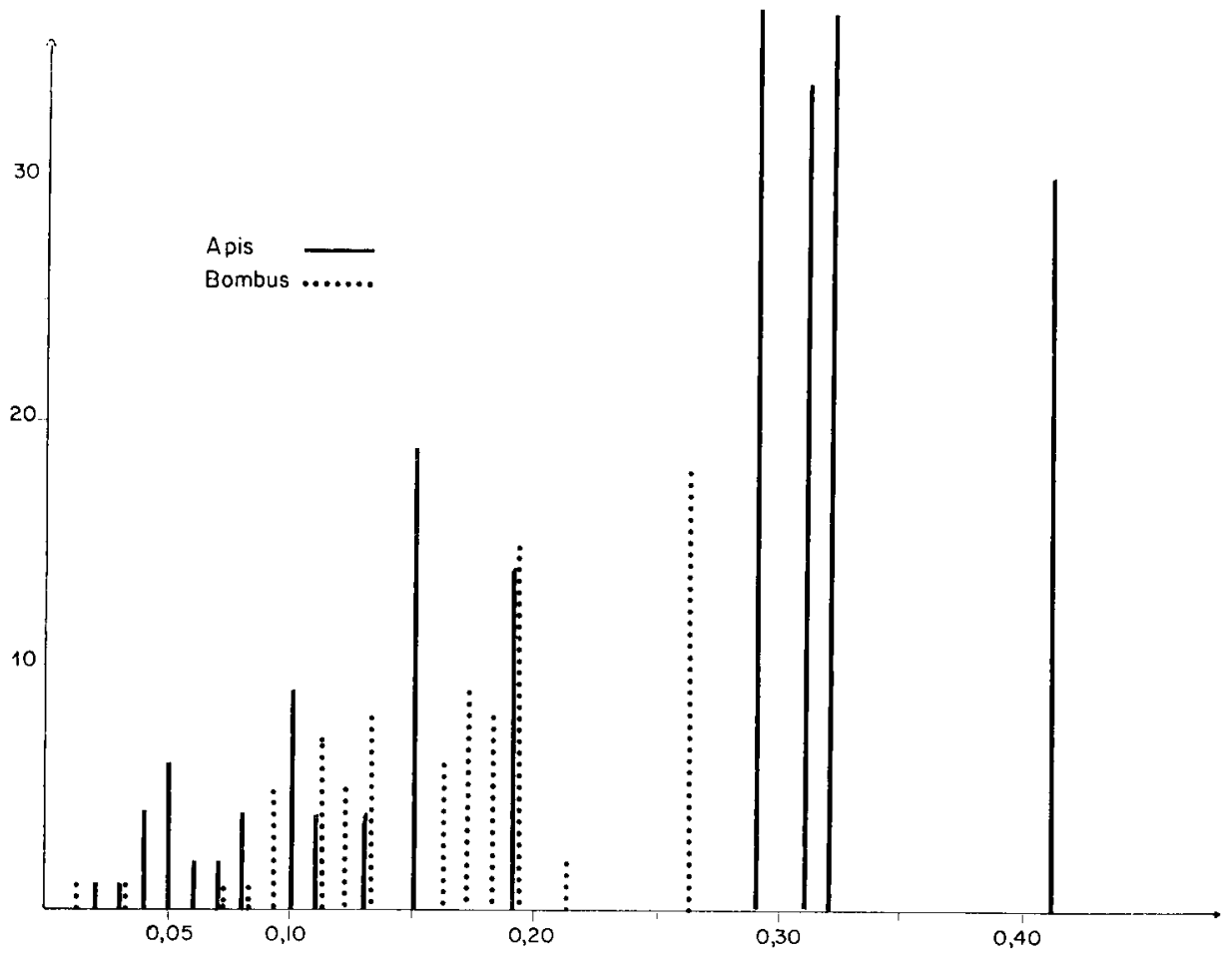

FIG. 1. - Nombre de couples monogénériques

Verticalement : nombre moyen de couples

Horizontalement : densité

Авв. 1. - Anzahl der Paare gleicher Art

Abszisse = Durchschnitszahl der Paare

Ordinate $=$ Dichte 
A densité égale, le nombre de couples d'abeilles est supérieur à celui des couples de bourdons. La différence, réduite aux faibles densités, s'accentue avec l'augmentation de la densité. De même, il est très rare de voir 3 bourdons butiner une fleur; tandis que cela arrive chez l'abeille : elles peuvent être 4 , voire 5 sur le même dahlia.

De plus, la densité moyenne maximum observée sur le champ est 0,26 pour les Bombus et 0,41 pour Apis. Il est important de rappeler ici que la taille moyenne d'un bourdon est supérieure à celle des abeilles. Cependant les ouvrières de Bombus sont de taille variable, et même les plus petites ne sont pas plus de 2 sur la même fleur.

On peut donc penser que les abeilles supportent mieux que les bourdons un butinage en commun.

Pour vérifier cela, j’ai confronté ces résultats à ceux qu'on obtiendrait si les animaux étaient indifférents les uns aux autres. Il faut donc calculer le nombre de couples observables si les insectes se rencontraient au hasard.

Pour ce faire, on calcule la probabilité pour qu'une fleur soit occupée par $0,1,2$ abeilles et/ou bourdons, en appliquant la formule suivante :

$$
P \mathbf{k}=\frac{(\mathrm{F}-1)^{\mathrm{I}-\mathrm{k}}}{\mathrm{F}^{\mathrm{I}}} \quad \frac{\mathrm{I}}{\mathbf{k}(\mathrm{I}-\mathbf{k})},
$$

dans laquelle

$\mathrm{Pk}=$ probabilité pour qu'une fleur soit occupée par k insectes,

$\mathrm{I}=$ nombre total d'insectes,

$\mathbf{F}=$ nombre total de fleurs,

$\mathbf{k}=$ nombre d'insectes sur une fleur.

On obtient ainsi

P0 = probabilité pour qu'une fleur soit libre.

$\mathrm{Pl}=$ probabilité pour qu'une fleur soit occupée par 1 insecte,

P2 = probabilité pour qu'une fleur soit occupée par 2 insectes,

On distingue alors l'occupation par les abeilles de celle par les bourdons en remplaçant I dans la formule Pk par Ia (nombre d'abeilles) ou Ib (nombre de bourdons).

La fréquence théorique est alors calculée :

$$
\mathrm{N}^{\prime} \text { calculé }=\mathrm{Pk} \times \mathbf{F} \text {. }
$$

On a ainsi calculé le nombre théorique d'insectes butinant seuls ou en couple mono ou hétérospécifique selon l'hypothèse de la rencontre au hasard.

Les figures 2 et 3 ( $\mathrm{N}^{\prime}$ fonction de $\mathrm{N}$ ) sont alors obtenus pour les Bombus et les Apis mellifica respectivement. 


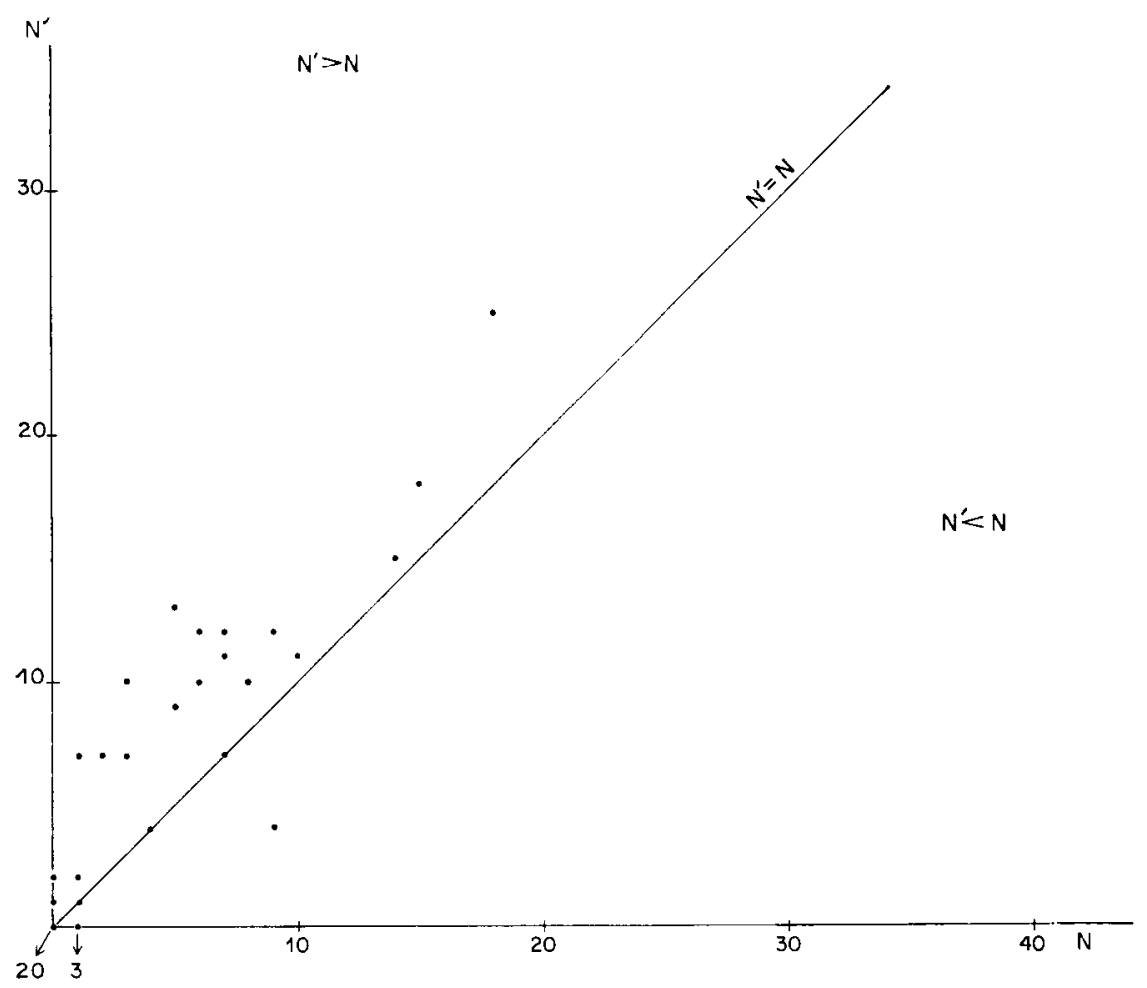

Fıg. 2. - Comparaison pour Bombus de $N^{\prime}$ calculê et de $N$ observé $\mathrm{N}^{\prime}<\mathrm{N}$ : attraction ; $\mathrm{N}^{\prime}=\mathrm{N}$ : indifférence; $\mathrm{N}^{\prime}>\mathrm{N}$ : répulsion Авв. 2. - Vergleich von $N^{\prime}$ berechnet und $N$ beobachtet für Bombus $\mathbf{N}^{\prime}<\mathbf{N}=$ Anziehung; $\mathbf{N}^{\prime}-\mathbf{N}=$ Indifferenz; $\mathbf{N}^{\prime}>\mathbf{N}=$ Abwehr

Si $N^{\prime}$ calculé est différent de $\mathbf{N}$ observé, c'est que les animaux ne sont pas indifférents les uns aux autres : quand $\mathrm{N}^{\prime}$ calculé $>\mathrm{N}$ observé il y a répulsion; quand $\mathrm{N}^{\prime}$ calculé $<\mathrm{N}$ observé il y a attraction.

Il apparaît nettement une différence de comportement entre les bourdons et l'abeille. Pour Bombus tous les points sont dans la zone $\mathrm{N}^{\prime}>\mathbf{N}$, tandis que pour Apis près de la moitié sont dans la zone $\mathrm{N}^{\prime}$ calculé $<\mathrm{N}$ observé. L'hypothèse se trouve donc confirmée par ce calcul.

Mais il faut rester prudent : l'outil mathématique utilisé ici n'autorise aucune affirmation. Simplement, on peut dire qu'il existe au cours du butinage, une certaine tendance à l'évitement chez Bombus au rapprochement chez Apis mellifica.

Cependant, malgré la présence de plus de 50 colonies d'abeilles à $200 \mathrm{~m}$ du champ d'observation, jamais il n'y eut autant d'abeilles que de fleurs épanouies dans le champ. 
Les densités maximales atteintes par les bourdons $(0,26)$ et les abeilles $(0,41)$ sont-elles donc des limites ? C'est possible, mais ces simples constatations ne permettent pas de le savoir. On peut seulement penser que la diminution du nombre de couples d'abeilles à la densité 0,41 par rapport à la densité 0,3 est un élément favorable à cette hypothèse : à cette forte densité, une répulsion commence peut-être à se manifester.

On peut aussi penser qu'une influence réciproque entre abeille et bourdon les empêche d'atteindre les densités limites naturelles. Apparemment il n'en est rien; en effet :

$1^{\circ}$ le nombre d'insectes volant sur le champ dépend surtout des conditions climatiques,

$2^{\circ}$ il n'y a pas diminution de la densité de bourdons quand celle des abeilles augmente, et inversement,

$3^{0}$ il y a des couples mixtes bourdon-abeille, dont le nombre semble lié aux densités des deux populations d'insectes (fig. 4),

$4^{\circ}$ on n'observe pas de combat entre bourdon et abeille sur les lieux de récolte.

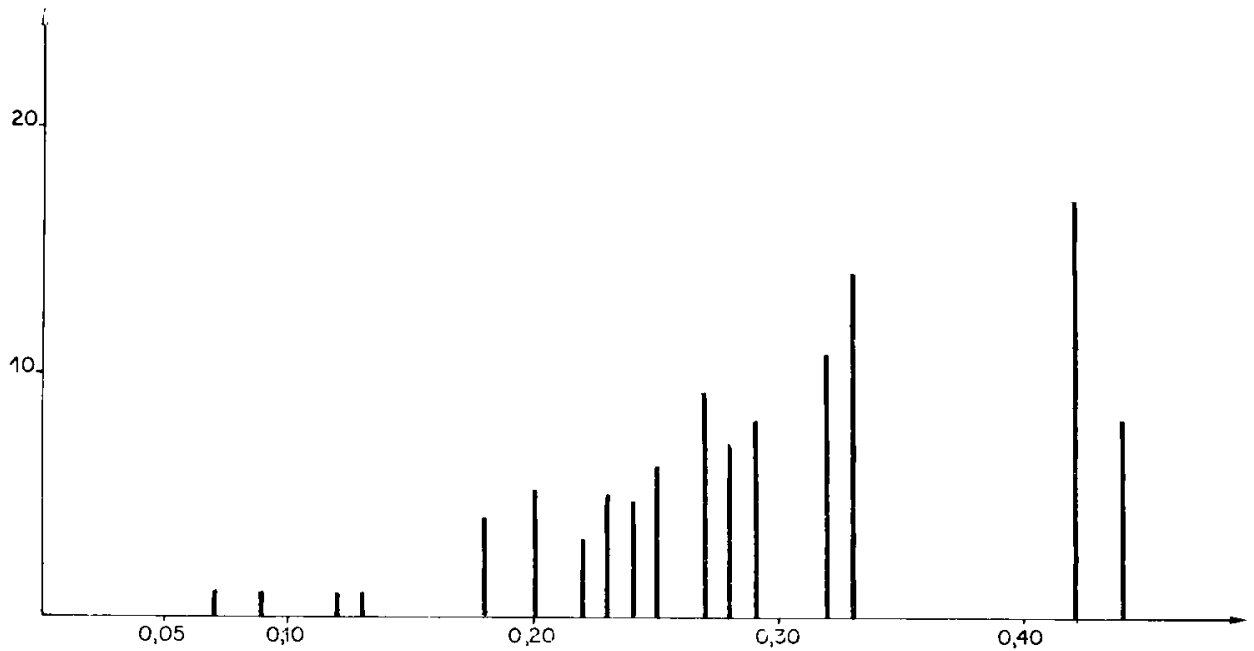

Fig. 1. - Nombre de couples mixtes Bombus-Apis mellifica

Verticalement : nombre moyen de couples

Horizontalement : densité

Aв8. 4. - Anzahl gemischter Paare von Bombus und Apis mellifica

Abszisse =- Durchschnittszahl der Paare

Ordinate $\cdots$ Dichte

Pourtant si on fait avec les couples mixtes Apis mellifica, Bombus terrestris le calcul fait avec les couples monogénériques, on constate une répulsion intergenre, $N^{\prime}$ calculé $>\mathrm{N}$ observé. Mais ici aussi, il faut rester très prudent (fig. 5). 


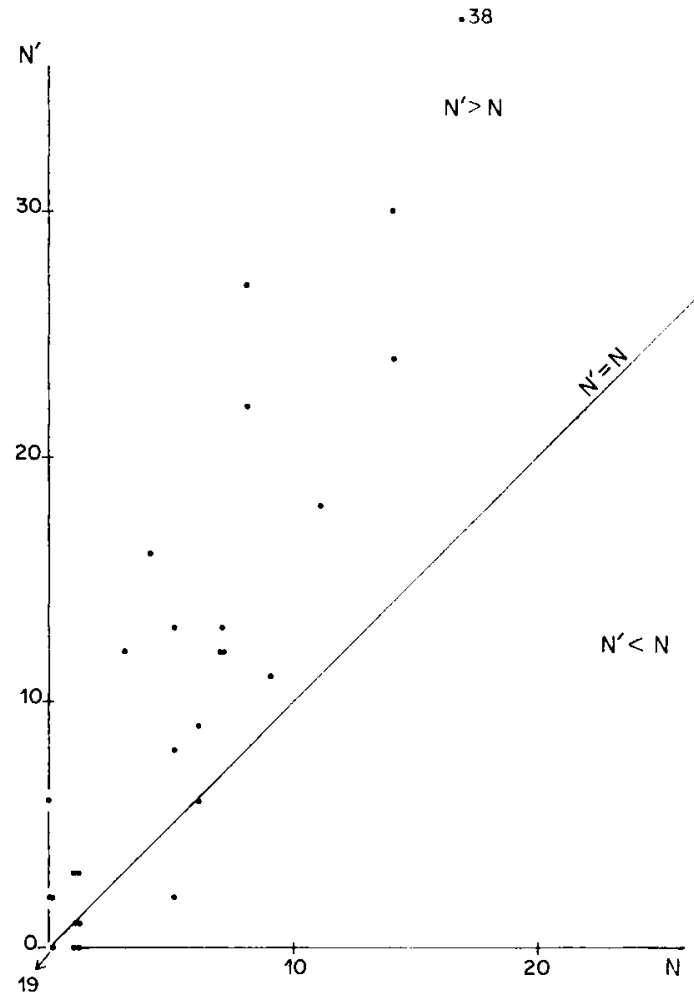

Fig. 5, - Comparaison pour les couples mixtes Bombus-Apis de $N^{\prime}$ calculè et de $N$ observé $\mathrm{N}^{\prime}<\mathrm{N}$ : attraction; $\mathbf{N}^{\prime}=\mathrm{N}$ : indifférence; $\mathrm{N}^{\prime}>\mathrm{N}$ : répulsion

Авв. 5. -Vergleich von $N^{\prime}$ berechnet und $N$ beobachtet für " gemischte Paare " von Bombus und Apis mellifica $\mathbf{N}^{\prime}<\mathbf{N}=$ Anziehung; $\mathbf{N}^{\prime}=\mathbf{N}=$ Indifferen $z ; \mathbf{N}^{\prime}>\mathbf{N}=$ Abwehr

En résumé, on peut dire que les Apis mellifica supportent mieux le butinage en commun que les Bombus. De plus, à ce niveau d'analyse, il semble que la rencontre entre deux individus soit facilitée par la présence de nombreux insectes sur le champ.

L'étude du comportement de chaque insecte amène à préciser cette proposition. Tout d'abord, la relation entre le nombre de rencontres (définies comme la réduction de l'écart entre deux individus à une distance $\leqslant 3 \mathrm{~cm}$ ) et la densité dépend des espèces considérées (tableau 1).

L'effet de la densité est net : plus une espèce est présente sur le champ, plus grande est la proportion de ses membres en rencontrant d'autres. Mais $B$. terrestris fait exception; cela peut se comprendre si l'on se rappelle que cette espèce est très agressive (BENEST 1972). 


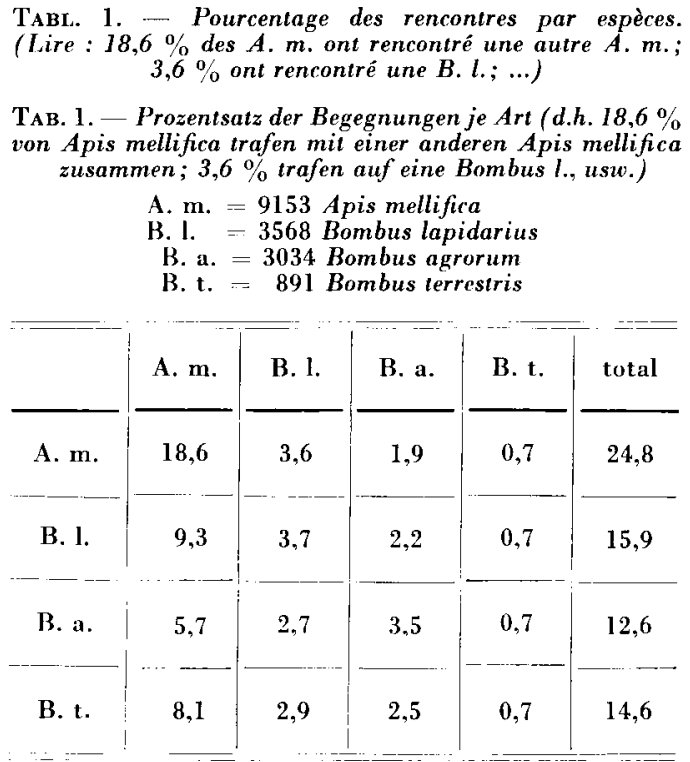

Le tableau 1 montre aussi que le choix de l'individu rencontré est en rapport avec le nombre de représentants de cette espèce : Apis mellifica et $B$. terrestris rencontrent tous deux plus d'abeilles que de B. lapidarius, ces derniers étant moins nombreux sur le champ, et ainsi de suite. Cependant, il semble s'opérer une sélection aussi en fonction de l'espèce de l'animal rencontré : Apis mellifica, B. agrorum et B. lapidarius rencontrent plus fréquemment des individus de même espèce qu'eux. Les B. terrestris sont trop peu nombreux : ils ne peuvent choisir, leurs rencontres se faisant au hasard, au prorata des densités.

Donc deux facteurs agissent ensemble et déterminent l'espèce de l'animal rencontré par un individu donné :

$1^{\circ}$ l'espèce de l'individu : Apis mellifica, Bombus lapidarius, B. agrorum rencontrent plus souvent des insectes de leur espèce. On peut penser qu'une certaine attraction se manifeste ainsi entre animaux de même espèce. Cependant, ce phénomène peut être masqué par l'influence du $2^{\mathrm{e}}$ facteur,

$2^{\circ}$ le nombre d'individus de cette espèce : plus une espèce est nombreuse sur le champ, plus elle est susceptible de rencontrer et d'être rencontrée par d'autres insectes.

Tel qu'il est défini plus haut, le terme " rencontre " ne présage en rien de son issue. Effectivement, on observe que les rencontres sont suivies soit d'un butinage en commun, soit plus rarement du départ de l'un et/ou l'autre insecte. 
Les rencontres sont peu nombreuses proportionnellement aux densités; mais quand elles se produisent, elles se terminent souvent par un butinage en commun, comme l'indiquent les valeurs du rapport :

$$
\frac{\text { nombre couples }}{\text { nombre rencontres }} \text { (tableau } 2 \text { ). }
$$

$$
\begin{gathered}
\text { nombre de couples } \\
\text { TABL. 2. - Rapport nombre dé rencontres } \\
\text { Même légende que le Tabl. I }
\end{gathered}
$$

\begin{tabular}{|c|c|c|c|c|}
\hline & A. $\mathrm{m}$. & B. 1. & B. a. & B. $t$. \\
\hline A. $\mathrm{m}$. & 92,71 & 89,25 & 78,85 & 83,57 \\
\hline- & -- & -- & $-\quad \ldots$ & - \\
\hline B. 1. & 89,25 & 90,97 & 75,60 & 92,30 \\
\hline B. a. & 78,85 & 75,60 & 77,98 & $65,2 \mathrm{I}$ \\
\hline B. $t$. & 83,57 & 92.3 & 65,21 & 100 \\
\hline
\end{tabular}

TAB. 2. - Verhältnis $\frac{\text { Anzahl Paare }}{\text { Zahl der Begegnungen }}$

Wie bei Tal. 1

Les différences sont faibles entre les différentes valeurs du rapport; l'influence des deux facteurs, densité et espèce, n'apparaît pas très importante : seul $B$. agrorum exprime une certaine préférence pour les bourdons de même espèce, compte tenu que l'abeille est très présente.

Une partie des rencontres se terminent par le départ de l'un ou des deux animaux.

La valeur du rapport

$$
\frac{\text { nombre de fuite }}{\text { nombre de non-fuite }}
$$

indique la tendance de l'animal occupant une fleur à la quitter à l'arrivée d'un congénère (tableau 3) quand un butinage en commun ne termine pas une rencontre.

L'occupant Apis mellifica ou B. agrorum laisse plus facilement la place à l'arrivant que $B$. terrestris. Ce dernier ne quitte la fleur que si l'arrivant est une abeille ou un $B$. agrorum. $B$. lapidarius se situe entre ces deux extrêmes.

Ici encore on observe un effet de la densité : on fuit plus un insecte dont l'espèce est bien représentée. Néanmoins, les bourdons fuient moins une 
abeille qu'un autre bourdon. De plus, B. lapidarius fuit encore moins à l'arrivée d'un autre $B$. lapidarius.

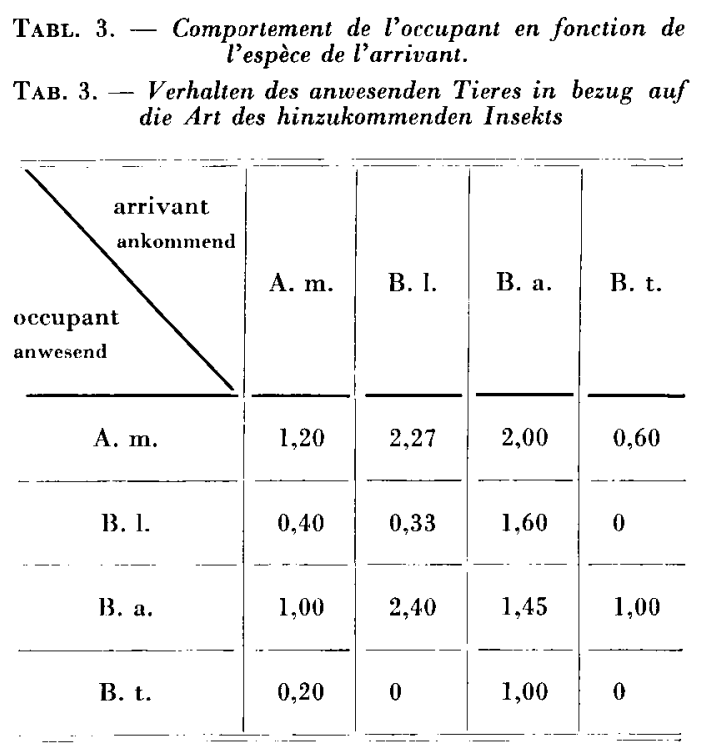

Ainsi l'arrivant ne chasse pas systématiquement l'occupant. La valeur du rapport

$$
\text { nombre de « s’éloigne » }
$$

nombre de c départ de l'occupant "

indique la tendance de l'arrivant à prendre la place de l'occupant (tableau 4).

Le comportement manifesté par l'arrivant dépend de l'espèce de l'occupant. Dans l'ensemble, l'arrivant chasse plus une butineuse Apis mellifica ou $B$. agrorum qu'une butineuse $B$. lapidarius ou $B$. terrestris dont il s'éloigne plus.

Il arrive qu'une rencontre se termine par le départ de l'arrivant et de l'occupant. B. lapidarius est la seule espèce qui, lors d'une rencontre intraspécifique ne laisse pas la fleur inoccupée : un seul des 2 animaux en présence s'éloigne; généralement, l'occupant reste en place : 3 fois sur 4 .

\section{DISCUSSION}

Rares sont les travaux étudiant les relations interindividuelles entre animaux butinant les mêmes fleurs. Brian (1957) a publié le premier travail concernant les bourdons sur ce problème. L'observation du butinage de fleurs artificielles par des ouvrières captives et de dahlias par des ouvrières libres 
confirme ce qui avait déjà été vu : la présence d'un congénère influence le comportement d'une butineuse. (BENEst, 1974).

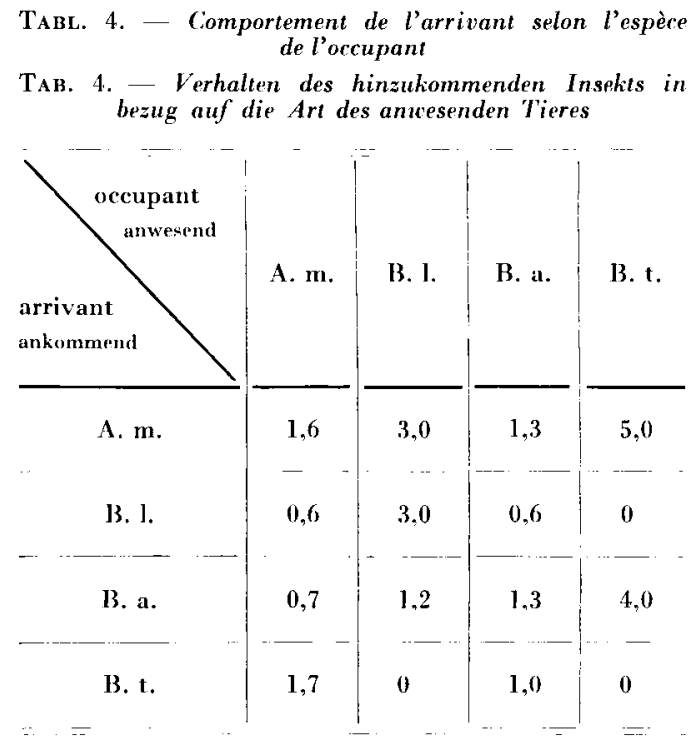

Ainsi les bourdons et les abeilles partagent plus volontiers une fleur avec un partenaire de même espèce que d'espèce différente. Deux facteurs semblent déterminer la quantité et la qualité des rapports entre deux espèces butinant un même champ : l'espèce à laquelle appartiennent les antagonistes et le nombre d'individus de ces espèces butinant le champ.

Par exemple dans les rapports intergénériques Bombus-Apis mellifica une compétition entre l'abeille et d'autres insectes a été montrée par PECK et Bolton (1946) et Pengelly (1954) pour qui l'abeille, par sa simple présence, écarte les autres pollinisateurs. BENEDEK et coll. (1971) observent même de Apis mellifica, quand leur densité est grande, attaquer les abeilles sauvages butinant le même champ de luzerne. Au contraire, Jones (1950) constate qu'il n'y a pas exclusion mutuelle entre l'abeille et les autres hyménoptères. Les résultats ne permettent pas de prendre position : selon le paramètre choisi, ils peuvent appuyer l'hypothèse de l'indifférence (variation des densités dans les populations) ou celle de l'évitement (nombre de couples mixtes).

Cependant on peut d'ores et déjà se poser la question des moyens utilisés par les butineuses pour se reconnaître.

Chez Apis mellifica Kalmus (1941) a montré l'importance des informations visuelles : leur propre image dans un miroir suffit à attirer les ouvrières. 
L'observation du butinage des dahlias indique que cela se produit aussi lors d'un butinage naturel. En effet les valeurs assez élevées du rapport

$$
\frac{\text { nombre de couples }}{\text { nombre de rencontres }} \text { (tableau 2) }
$$

amènent à penser que ce ( choix » du partenaire de butinage est fait avant que l'animal se pose. Comment? Il paraît logique que la vision soit impliquée : en effet, on remarque qu'un insecte reste plus souvent avec un autre de même couleur que lui. Ainsi $B$. lapidarius butine le plus fréquemment avec $B$. terrestris ou des congénères de même espèce : ils sont tous les deux de couleur principalement noire. De même $B$. agrorum reste le plus souvent avec $B$. agrorum ou Apis mellifica : tous deux sont jaunes.

Remarque : le faible nombre d'observations pour cette espèce est probablement responsable $d u$ fait que toutes les rencontres entre $2 B$. terrestris se terminent par un butinage en commun.

Cette hypothèse est appuyée par le fait suivant : le taux de rencontres par rapport à la densité et le taux de couples par rapport au nombre de rencontres est supérieur pour des individus de couleurs relativement proches que pour des insectes de couleurs différentes (tableau 5).

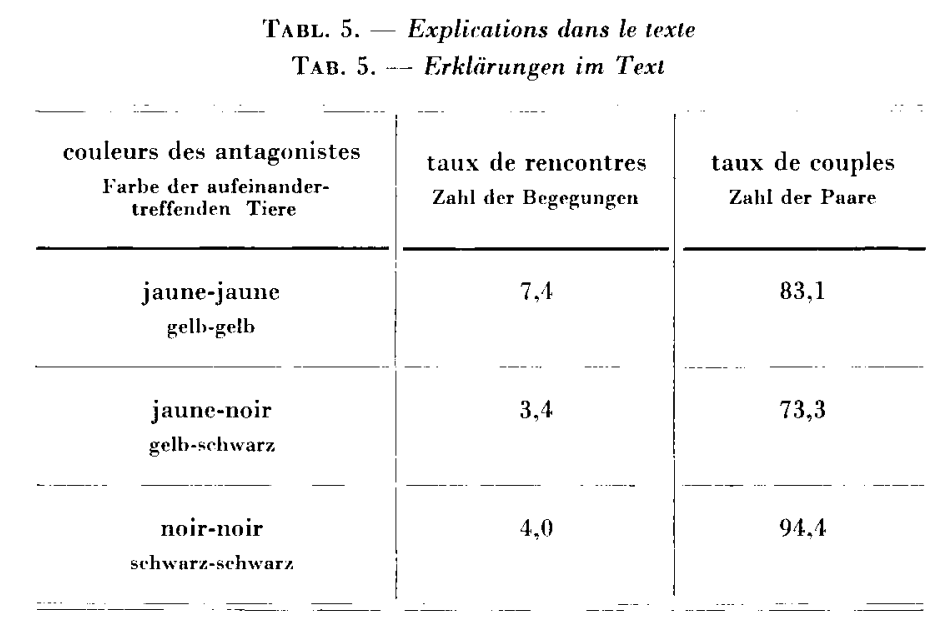

En fait il conviendrait aussi peut-être de préciser la colonie d'origine : en effet, Johnson et Hubbes (1974) montrent qu'au lieu de récolte Trigona sylvestriana et $T$. corvina distinguent les membres de leur nid des autres congénères.

Plusieurs auteurs (Kalmus 1941, Lecomte 1955, Weaver 1957) ont observé des comportements agressifs entre des ouvrières d'Apis mellifica butinant des nourrisseurs ou des fleurs. Je n'en ai jamais vu chez Bombus; mais on sait que bourdons et abeilles, bien que phylogénétiquement proches, pré- 
sentent de nombreuses différences; en particulier, j’ai montré que Bombus défend plus son nid contre des intrus d'espèces différentes que contre ceux de même espèce : les observations rapportées ici montrent que cela est aussi valable pour les lieux de récolte de nourriture, confirmant ce que Brian avait déjà écrit (1957).

Ainsi il apparaît que les relations entre deux individus butineurs résultent de l'équilibre entre deux tendances qui s'opposent une tendance au rapprochement, une tendance à l'éloignement. Plusieurs facteurs, dont quelques-uns sont étudiés ici, vont intervenir qui favoriseront l'une ou l'autre.

$$
\begin{array}{r}
\text { Reçu pour publication en février } 1976 . \\
\text { Eingegangen im Februar } 1976 .
\end{array}
$$

\section{ZUSAMMENFASSUNG}

An den Sammelplätzen bestehen zwischen den sammelnden Insekten entweder interspezifische oder intraspezifische Beziehungen. Die Kontaktaufnahme zwischen den Tieren kann direkt oder indirekt erfolgen.

Unmittelbare Kontakte werden an Apis mellifica und Bombus lapidarius, B. terrestris und $B$. agrorum beobachtet, die frei auf einem $472 \mathrm{~m}^{2}$ grossen Zwergdahlien-Feld sammeln. Die Quantitätsbestimmung beruht auf den Begegnungen der Insekten. Fs wird festgestellt, dass die Zahl der interspezifischen zu der der intraspezifischen Begegnungen proportional dem Verhältnis von Insektenzahl zu Blütenzahl ist (Abb. 1,4), was darauf hinweisen könnte, dass die Insektenbegegnungen rein zufällig erfolgen. Die Berechnung der tatsächlich zufälligen Begegnungen zeigt aber, dass bei den Bienenarbeiterinnen vielmehr eine gegenseitige Anziehung besteht, und dass die Hummeln einander eher abstossen; ferner, dass Bienen und Hummeln zu gegenseitiger Abwehr neigen. (Abb. 2, 3, 5).

Eine genauere Analyse ergibt für die Hummeln eine gegenseitige intraspezi fische Duldung und ein interspezifisches Abwehren des Partners. Der Partner wird visuell erkannt. Diese Hypothese stützt sich auf die Tatsache, dass die Tiere einander auf eine Entfernung von mehr als $3 \mathrm{~cm}$ erkennen, sowie darauf, dass Insekten gleicher Farbe eher geduldet werden als solche mit anderer Färbung (Tab. 5).

Schliesslich tritt ein deutlicher Unterschied zwischen Bombus und Apis mellifica zutage : die Bienen ertragen das Sammeln in der Gemeinschaft besser als die Hummeln.

\section{REFÉRENCES BIBLIOGRAPHIQUES}

Benedex P., Manninger S., Devai G., 1971. Expériences sur la pollinisation de la luzerne et du trètle incarnat par Apis mellifica. Novenytermeles 20, 2 : 145-156 (Hongrois).

Benest G., 1972. Note préliminaire sur la réaction d'ouvrières de Bombus terrestris à la présence d'intruses. Insectes soc., 19 (2), 131-135.

Benest G., 1974. Contribution à l'étude des relations interindividuelles chez quelques Bombus, 76 p. ('Thèse $3^{\text {e }}$ cycle Sci. environ. comportment. Rennes. 1974).

Brian A.D., 1957. Differences in the flowers visited by four species of Bombus. J. anim. Ecol., $26,71-98$. 
Johnson L.K., Hubbel S.P., 1974. Aggression and competition among stingless bees : field studies. Ecology, 55, 120-127.

Jones L.M., 1950. Report 12th alfalfa improvement congr., 38-40. Report 12th alfalfa.

Kalmus H., 1941. The defence of a source of food by honeybees. Nature, Lond., 148-151.

Lecomte J., 1955. Observations sur le comportement d'abeilles vivant en serres chaudes durant l'hiver. L'apiculteur, 39-42.

Lecomte J., 1961. Le comportement agressif des ouvrières d'Apis mellifica. Thèse Sci. nat. Paris, 1963 in : Ann. Abeille $4: 165-270$.

Leconte J., 1963. Étude des échanges de nourriture de la colonie de Bourdons au moyen de radioisotopes. C. $R$. Acad. Sci., 257, 3664-3665.

Peck O., Bolton J.L., 1946. Sci. Agr., 26, 388-417.

Pengelly D.H., 1954. 48th Annu. Rept. Entomol. Soc. Ontario, 101-118.

WEAver N., 1957. The foraging behaviour of honeybees on hairyvetch. II. The foraging area and the foraging speed. Insectes sociaux, 4, 44-57. 